\title{
Increasing of surface quality in friction free-form surfaces of knee prosthesis
}

\author{
J. Chaves-Jacob, J.M. Linares (2)*, J.M. Sprauel \\ ISM UMR6233, Aix Marseille University, Avenue Gaston Berger, 13625 Aix en Provence Cedex 1, France
}

A B S T R A C T

\begin{abstract}
Keywords: $\quad$ This paper deals with increasing of the quality of knee prosthesis friction surfaces, in order to reduce wear discontinuities which lead to imperfections on machined surfaces. A new five degree polynomial Surface interpolation method has therefore been developed to limit such defect. A Design of Experiment has then been carried out to optimize the discretisation intervals of the CAD/CAM model and to test the capacity of Roughness this new strategy to smooth undesired typical geometrical discontinuities. The method is also compared to standard techniques. The results show a significant improvement of the surface quality provided by the new polynomial interpolation.
\end{abstract}

\section{Introduction}

To reduce wear debris production, friction surfaces of knee prostheses require extremely high quality. In the manufacturing phase, data file format conversion (IGES, STEP, ...) is often used to transfer the CAD model to the CAM system. Undesired geometrical discontinuities can thus be engendered on friction surfaces. A discontinuity in tangency $\left(\mathrm{C}^{1}\right)$ of an industrial knee prosthesis CAD model is illustrated in Fig. 1. During machining, the toolpath computed for such imperfection will induce smoothing errors and generate marks on the prosthesis surface. Nevelos [1] has studied the wear mechanisms of knee prostheses and demonstrated the large impact of such defect. The influence of discontinuities on wear debris production is illustrated in Fig. 1.

A great number of works have been already carried out to increase the quality of machined biomedical free-form surfaces. An exhaustive synthesis of this topic has been presented in a CIRP keynote paper of 2007 [2]. Most research tasks studied the efficiency of a toolpath generation method to smooth CAD models without introducing significant error. For that purpose manufacturing of free-form surfaces has already been realised using BSpline toolpath interpolations. Lartigue et al. 3] proposed such a solution based on computations of two toolpaths derived from two different points on each tool axis. The authors showed the usefulness of this method to decrease surface faceting. Another technique has also been proposed by Schützer et al. [4]. In this method, contrary to classical CAM systems, the B-Spline toolpath is directly derived from the CAD model data and does not use a faceting approximation of the surface. Surface quality is also greatly influenced by machining feed rate. This problem has been studied by Altintas et al. [5] who optimized the feed rate modulation along the B-Spline toolpath. The aim of our work is now to recover the quality of surfaces already defined by toolpaths derived from CAD/CAM systems which however introduced

\footnotetext{
* Corresponding author.
}

undesired discontinuities. For that purpose, a new five degree polynomial interpolation method is developed to smooth the imperfections. Typical functional parameters will be selected to quantify the surface quality. These indicators will be evaluated through optical roughness measurements. Surfaces realised through polynomial interpolation will thus be characterised and compared to the same ones obtained with a classical smoothing technique already incorporated in CNC facilities. This last procedure is based on linear segments whose edges are rounded to obtain continuous slopes. It will be named enhanced linear interpolation. A Design of Experiment (D.O.E.) will be used to compare the two techniques. The D.O.E. will also result in an empirical model which allows predicting the surface quality and optimizing the discretisation intervals of the CAD/CAM model. This model will be tested on a real knee prosthesis surface.

\section{Surface quality of knee prostheses}

A high level of quality is needed on friction surfaces of knee prostheses. Now, the surface quality mainly depends on the toolpath used for machining and the associated interpolations. In the next subsection, a new polynomial interpolation method is therefore proposed to improve the quality of biomedical free-form surfaces.

\subsection{Presentation of the polynomial interpolation method}

To increase the mathematical continuity of the final tool trajectory, successive points of the toolpath are interpolated by sliding five degree polynomials. Contrary to standard solutions, this method ensures continuity in slope and curvature over the whole toolpath, smooth acceleration variations and does not induce any oscillation. As presented in Fig. 2, the stability of the method is obtained thanks to the use of intermediate parabolas which allow evaluating the slope and curvature at each point of the toolpath. For that purpose, the slope and curvature of each point $M_{i}$ are respectively approximated by the first and second order 


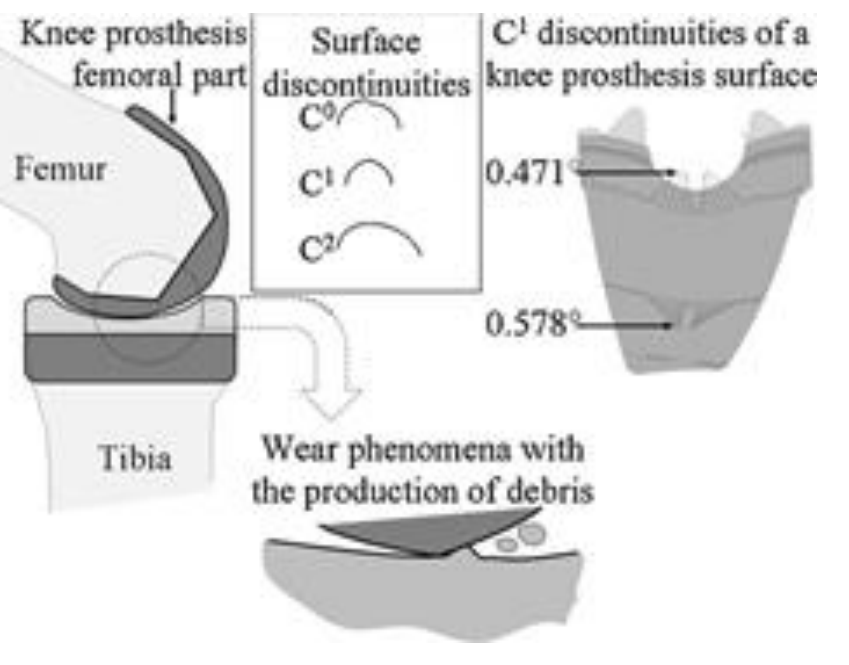

Fig. 1. Influence of surface discontinuities on the wear of knee prostheses.

derivatives of the parabola which fits the triplet $\left(\mathrm{M}_{\mathrm{i} \ddot{y}}, \mathrm{M}_{\mathrm{i}}, \mathrm{M}_{\mathrm{i}+\mathrm{i}}\right)$. The coefficients of the five degree polynomial interpolating two given successive toolpath points is then deduced from the coordinates, slopes and curvatures imposed at the boundaries of the smoothing interval.

\subsection{Definition of the Design of Experiment}

Three main misfits are considered in our D.O.E.: discontinuity in position $\left(\mathrm{C}^{0}\right)$, in slope $\left(\mathrm{C}^{1}\right)$ and in curvature $\left(\mathrm{C}^{2}\right)$. Our interpolation method is therefore tested on the special workpiece, presented in Fig. 3, since it contains all these types of imperfections. Two factors are considered in the D.O.E.: the amplitude of the discontinuities, expressed versus a unique dimensionless ratio $\mathrm{H}$, and the Interval of Tolerance (IT illustrated in Fig. 2) selected to compute the linear toolpath. Fig. 3 gives the relation between ratio $\mathrm{H}$ and the different misfit amplitudes. The same toolpath points are used for both, the linear and polynomial interpolation techniques. Table 1 presents

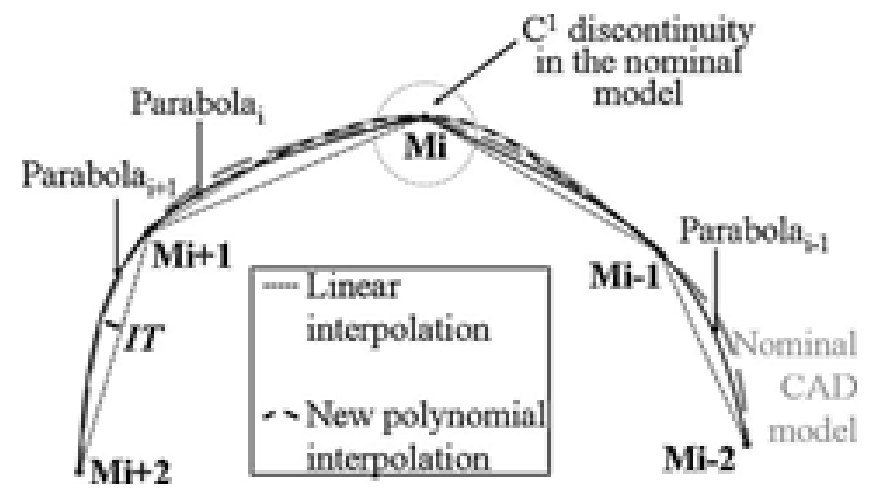

\begin{tabular}{l} 
I Fig. 2. Five degree polynomial interpolation method. \\
\begin{tabular}{|c|c|c|c|}
\hline$H$ & $\mathrm{C}^{\circ}(\mathrm{mm})$ & $\left.\mathrm{C}^{1}()^{\circ}\right)$ & $\mathrm{C}^{2}\left(\mathrm{~mm}^{-1}\right)$ \\
\hline 0.001 & 0.001 & 0.115 & 0.0006 \\
\hline 0.005 & 0.005 & 0.573 & 0.03 \\
\hline 0.015 & 0.015 & 1.719 & 0.08 \\
\hline 0.025 & 0.025 & 2.865 & 0.12 \\
\hline 0.029 & 0.029 & 3.324 & 0.125 \\
\hline
\end{tabular} \\
\hline
\end{tabular}

Fig. 3. Workpiece on 5-axis CNC machine.
Table 1

Experimental parameters (IT, $\mathrm{H})$ and related normalised factors $\left(\mathrm{X}_{1}, \mathrm{X}_{2}\right)$

\begin{tabular}{lrlr}
\hline IT $(\mathrm{mm})$ & $\mathrm{X}_{1}$ & $\mathrm{H}$ & $\mathrm{X}_{2}$ \\
\hline 0.005 & $\ddot{y} 1$ & 0.005 & $\ddot{y} 1$ \\
0.055 & 1 & 0.005 & $\ddot{y} 1$ \\
0.005 & $\ddot{y} 1$ & 0.025 & 1 \\
0.055 & 1 & 0.025 & 1 \\
0.03 & 0 & 0.015 & $\mathrm{C}$ \\
0.03 & 0 & 0.015 & $\mathrm{C}$ \\
0.03 & 0 & 0.015 & 0 \\
\hline
\end{tabular}

the seven experiments realised for each method. Normalised parameters $X_{1}$ and $X_{2}$, defined by Eq. (1), are then built to express the D.O.E. model

$$
\begin{aligned}
& \gtrless_{X_{1}} 1 / 4 \frac{2 \text { IT } \ddot{y} \text { 00:055 p 0:005p }}{0: 055 \ddot{y} 0: 005} \\
& \mathrm{X}_{2} \frac{2 \mathrm{H} \ddot{\mathrm{y}} \mathrm{y} 0: 025 \mathrm{p} 0: 005 \mathrm{p}}{0: 025 \ddot{y} 0: 005}
\end{aligned}
$$

These experimental factors are fixed to the corners and centre of the scanned domain. A double repetition is performed at the centre. The machining tests are carried out using the same regular cutting parameters for all the experiments.

\subsection{Definition of the surface parameters}

After machining, the realised surfaces are accurately characterised through optical measurements using the chromatic confocal sensing CMM, shown in Fig. 4. The resolution of this $\mathrm{CMM}$ is about $10 \mathrm{~nm}$. The measurements are carried out on areas of $2.5 \mathrm{~mm} 11 \mathrm{~mm}$, centred on each nominal discontinuity. The length of these zones is set to the direction of the tool feed and scanned with a step of $4 \mathrm{~mm}$. In relation to the radial depth of cut $(0.2 \mathrm{~mm})$, a $20 \mathrm{~mm}$ step is used in the width, leading to 10 measured lines by pass.

The first response selected to qualify each experiment is the average deviation (AD). It is obtained by subtracting the nominal profile of the $\mathrm{CAD}$ model from the measured coordinates. It characterises the deviations from the $\mathrm{CAD}$ model. The response $\mathrm{AD}$ is finally defined as follows:

$D^{1 / 4}{ }_{A}^{1}{ }_{A}^{Z Z Z} Z_{M} \partial x ; y P \ddot{y} Z_{C A D} \partial x ; y p j d x d y$

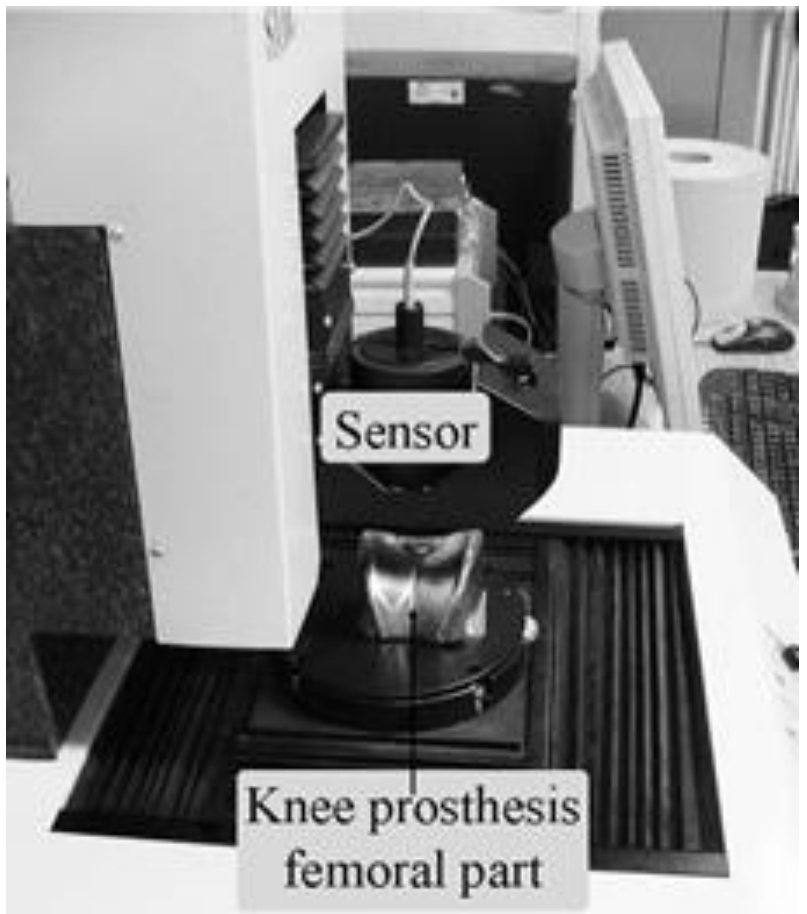

Fig. 4. Optical easumements using a chromatic confocal sensing CMM. 


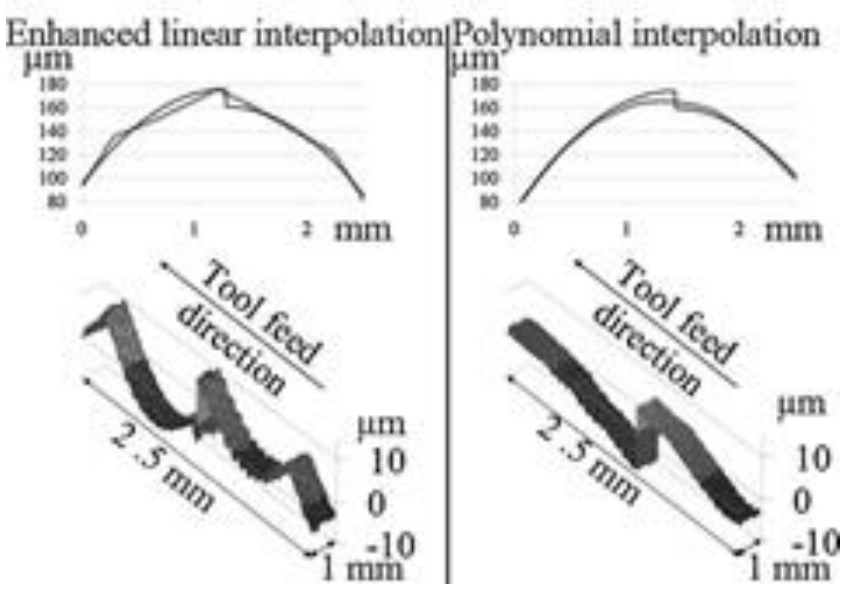

Fig. 5. Residues of subtraction of nominal surface (discontinuity in $\mathrm{C}^{0}$ )

with: A the measured area (here $\left.2.5 \mathrm{~mm}^{2}\right), \mathrm{Z}_{\mathrm{M}}(\mathrm{x}, \mathrm{y})$ the measured altitude and $Z_{C A D}(x, y)$ the $C A D$ profile as fitted to the acquired data by least squares. The result of such calculation is illustrated in Fig. 5 showing a 2D section of the scanned area. The initial discontinuity of the CAD model re-emerges in the computed residues.

The second response selected to qualify the experiments is the smoothness $(\mathrm{Sm})$. It expresses the residue between the measured points and a smooth profile which would satisfy the functional requirements of biomedical friction surfaces. The smooth surface is defined by a set of cubic spline polynomials complying with the continuity in $\mathrm{C}^{0}, \mathrm{C}^{1}$ and $\mathrm{C}^{2}$ and fitted to the acquired data by least squares. The response $\mathrm{Sm}$ is finally defined as follows:

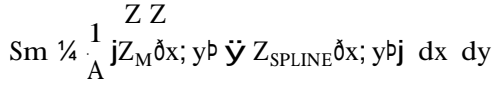

with: $Z_{\text {SPLINE }}(x, y)$ the smooth spline profile. Fig. 6 shows a $2 D$ section of such smooth surface and the calculated residues.

\subsection{Model of experiments}

In a first time, the surface quality is expressed as a simple multilinear model (Eq. (4))

$\mathrm{Y}^{1 / 4} \mathrm{~b}_{0} \mathrm{p} \mathrm{b}_{1} \mathrm{X}_{1} \mathrm{p} \mathrm{b}_{2} \mathrm{X}_{2} \mathrm{p} \mathrm{b}_{12} \mathrm{X}_{1} \mathrm{X}_{2}$

The effects $\left(b_{1}\right.$ and $\left.b_{2}\right)$ of the two factors $\left(X_{1}, X_{2}\right)$ and the interaction $\left(b_{12}\right)$ are computed by least squares optimization. Table 2 gives the functions ( $\mathrm{AD}$ and $\mathrm{Sm}$ ) evaluated for the three types of discontinuity and the two interpolation methods. The coefficients

\section{Test with $I T=0.055 \mathrm{~mm}$ and $H=0.015$}

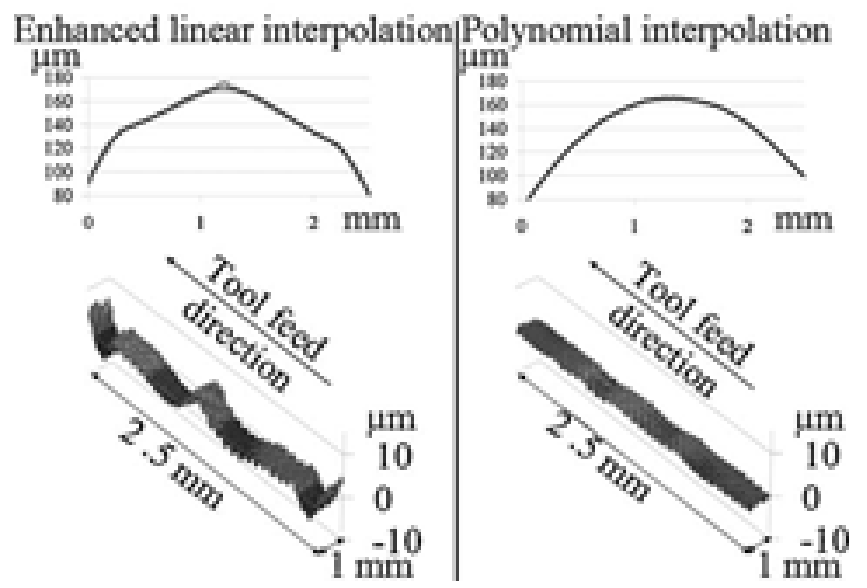

Table 2

AD and Sm models derived from our experiments.

\begin{tabular}{cccc}
\hline & Enhanced linear interpolation & Polynomial interpolation \\
\hline $\mathrm{AD}$ & $\mathrm{C}^{0}$ & $3.52+1.62 \mathrm{X}_{1}+0.65 \mathrm{X}_{2} \ddot{\mathrm{Y}} 0.01 \mathrm{X}_{1} \mathrm{X}_{2}$ & $2.63+0.85 \mathrm{X}_{1}+1.31 \mathrm{X}_{2}+0.71 \mathrm{X}_{1} \mathrm{X}_{2}$ \\
$\mathrm{AD}$ & $\mathrm{C}^{1}$ & $3.00+2.32 \mathrm{X}_{1} \ddot{\mathrm{Y}} 0.15 \mathrm{X}_{2} \ddot{\mathrm{y}} 0.26 \mathrm{X}_{1} \mathrm{X}_{2}$ & $1.33+0.40 \mathrm{X}_{1}+0.24 \mathrm{X}_{2}+0.16 \mathrm{X}_{1} \mathrm{X}_{2}$ \\
$\mathrm{AD}$ & $\mathrm{C}^{2}$ & $3.56+2.74 \mathrm{X}_{1}+0.44 \mathrm{X}_{2}+0.57 \mathrm{X}_{1} \mathrm{X}_{2}$ & $1.53+1.35 \mathrm{X}_{1}+1.41 \mathrm{X}_{2}+1.43 \mathrm{X}_{1} \mathrm{X}_{2}$ \\
$\mathrm{Sm}$ & $\mathrm{C}^{0}$ & $1.30+0.17 \mathrm{X}_{1}+0.11 \mathrm{X}_{2}+0.04 \mathrm{X}_{1} \mathrm{X}_{2}$ & $0.73 \ddot{\mathrm{y}} 0.32 \mathrm{X}_{1}+0.32 \mathrm{X}_{2} \ddot{\mathrm{y}} 0.32 \mathrm{X}_{1} \mathrm{X}_{2}$ \\
$\mathrm{Sm}$ & $\mathrm{C}^{1}$ & $0.88+0.06 \mathrm{X}_{1} \ddot{\mathrm{y}} 0.03 \mathrm{X}_{2} \ddot{\mathrm{y}} 0.08 \mathrm{X}_{1} \mathrm{X}_{2}$ & $0.57 \ddot{\mathrm{y}} 0.07 \mathrm{X}_{1}+0.04 \mathrm{X}_{2} \ddot{\mathrm{y}} 0.01 \mathrm{X}_{1} \mathrm{X}_{2}$ \\
$\mathrm{Sm}$ & $\mathrm{C}^{2}$ & $1.24+0.31 \mathrm{X}_{1}+0.22 \mathrm{X}_{2}+0.11 \mathrm{X}_{1} \mathrm{X}_{2}$ & $0.64+0.04 \mathrm{X}_{1}+0.19 \mathrm{X}_{2}+0.05 \mathrm{X}_{1} \mathrm{X}_{2}$ \\
\hline
\end{tabular}

of the average deviation (AD) function and the error bars calculated for two standard deviations are also presented in the graphics of Fig. 7.

For both interpolation methods, the two factors (IT and $\mathrm{H}$ ) have larger effects onto the average deviation than to the smoothness. As expected polynomial interpolation results in a better surface quality than the classical enhanced linear interpolation. Polynomial interpolation leads however to significant interaction between the two factors.

\subsection{Comparison of the two interpolation methods}

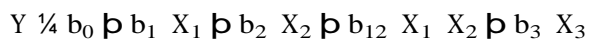

As already pointed out, polynomial interpolation seems more efficient than the linear one. To accurately compare the two techniques, a factor, $X_{3}$, is now added to the model to account for the employed strategy (linear or polynomial interpolation). Fig. 8 defines the values of $X_{1}, X_{2}$ and $X_{3}$ for this D.O.E. Coefficient $b_{3}$ representing the effect of the selected strategy onto the measured responses is also presented. The results demonstrate that the polynomial method reduces significantly the average deviation $(\mathrm{AD})$ and the smoothness $(\mathrm{Sm})$ of the manufactured surfaces. For a discontinuity in $\mathrm{C}^{2}$ and in comparison to linear interpolation, polynomial interpolation leads to reduce the smoothness of about $1 \mathrm{~mm}$.

\section{Optimization of the surface quality}

Our polynomial smoothing technique proved thus to be significantly more efficient than the classical enhanced linear interpolation to obtain surfaces of high quality. The aim of this section is now to determine an accurate empirical model permitting to optimize the discretisation intervals of the CAD/ CAM model. The model will account for the two factors IT and $\mathrm{H}$. However, the average deviation (AD) will no longer be studied since it integrates a part of the initial undesired discontinuities. In fact, as shown by the example of Fig. 6, machining performed with polynomial interpolation led to significant smoothing of $\mathrm{C}^{\mathrm{O}}$ discontinuities while deviations to the nominal profile are still remaining (Fig. 5).

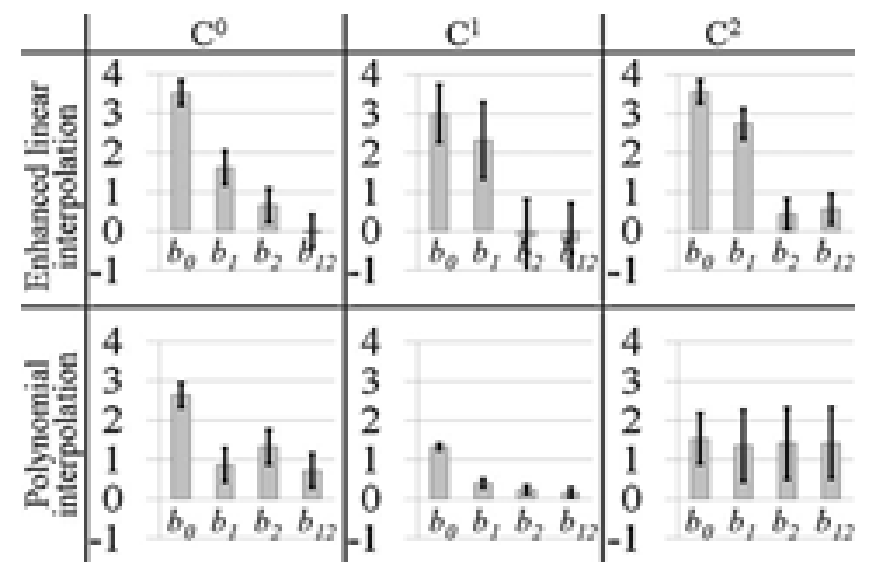




\begin{tabular}{|c|c|c|c|c|c|}
\hline$I T$ & $X_{1}$ & $H$ & $X_{2}$ & strategy & $X_{3}$ \\
\hline 0.005 & -1 & 0.005 & -1 & $\mathrm{G}_{1}$ & -1 \\
\hline 0.055 & 1 & 0.005 & -1 & $\mathrm{G}_{1}$ & -1 \\
\hline 0.005 & -1 & 0.025 & 1 & $\mathrm{G}_{1}$ & -1 \\
\hline 0.055 & 1 & 0.025 & 1 & $\mathrm{G}_{1}$ & -1 \\
\hline 0.03 & 0 & 0.015 & 0 & $\mathrm{G}_{1}$ & -1 \\
\hline 0.03 & 0 & 0.015 & 0 & $\mathrm{G}_{1}$ & -1 \\
\hline 0.03 & 0 & 0.015 & 0 & $\mathrm{G}_{1}$ & -1 \\
\hline 0.005 & -1 & 0.005 & -1 & POLY & 1 \\
\hline 0.055 & 1 & 0.005 & -1 & POLY & 1 \\
\hline 0.005 & -1 & 0.025 & 1 & POLY & 1 \\
\hline 0.055 & 1 & 0.025 & 1 & POLY & 1 \\
\hline 0.03 & 0 & 0.015 & 0 & POLY & 1 \\
\hline 0.03 & 0 & 0.015 & 0 & POLY & 1 \\
\hline 0.03 & 0 & 0.015 & 0 & POLY & 1 \\
\hline
\end{tabular}

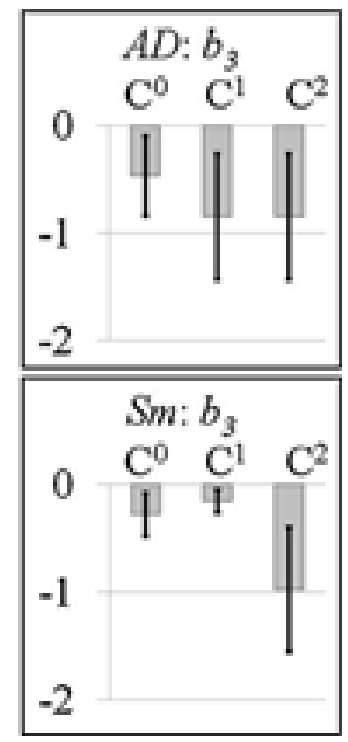

Fig. 8. D.O.E. to compare the two interpolation methods.

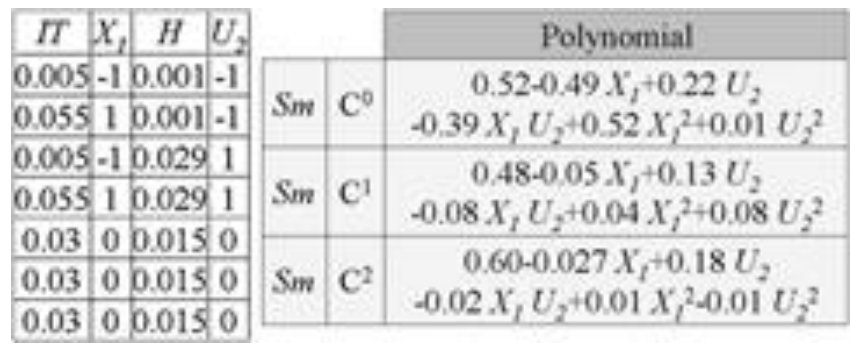

Fig. 9. Accurate models for polynomial interpolation.

\subsection{Model}

To increase the experimental domain of $\mathrm{H}$, its range has been extended to [0.001, 0.029]. Thus, a new parameter, $U_{2}$ (Eq. (6)), is introduced to be substituted to $\mathrm{X}_{2}$. The new non linear model presented in Eq. (7) is also selected to find an accurate model. Fig. 9 presents the values of the experimental factors and the obtained models

$\mathrm{U}_{2} 1 / 4 \frac{2 \mathrm{H} \ddot{\mathrm{y}} \text { 00:029 p 0:001p }}{0: 029 \ddot{y} \text { 0:001 }}$

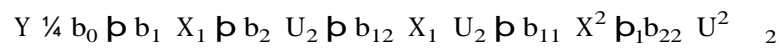

The smoothness parameter for a discontinuity in $\mathrm{C}^{1}$ is also detailed. Indeed, this configuration frequently occurs in the production of prosthesis friction surfaces. Fig. 10 illustrates the model obtained for such imperfection and its statistical limit envelop computed for two standard deviations [6].

\subsection{Experimental validation}

To validate the developed tools (five degree polynomial interpolation and empirical predictive model), friction surfaces of a femoral knee prosthesis have been machined. The manufactured workpiece can be observed in Fig. 4. Continuity in $\mathrm{C}^{\mathrm{O}}$ is assured between the eight parts of the friction surface but $\mathrm{C}^{1}$ discontinuities still exist in the CAD model of the prosthesis. The polynomial interpolation strategy has been used to realise this surface.

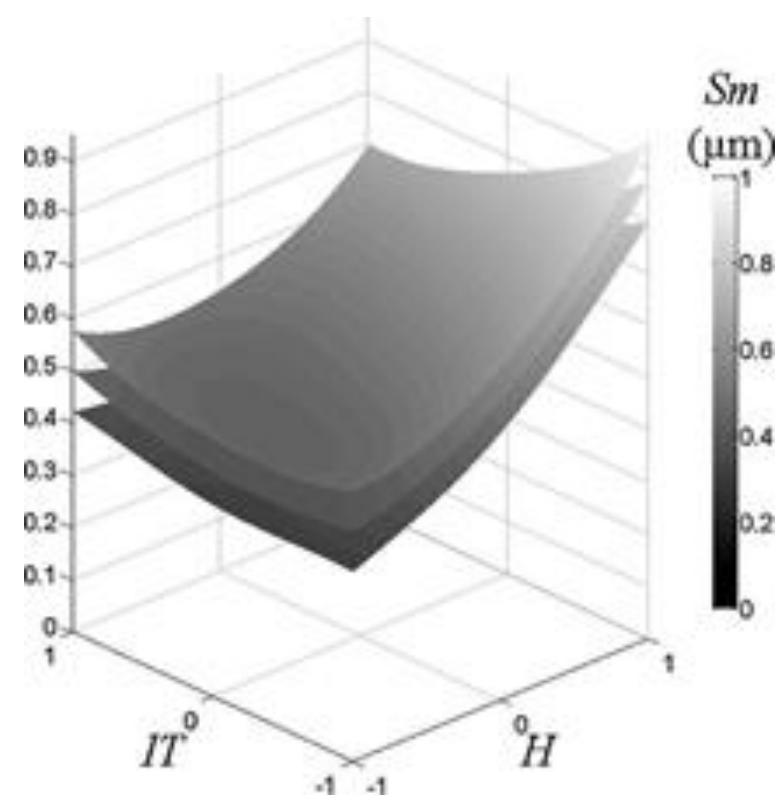

Fig. 10. Sm model of a $C^{1}$ discontinuity.

The Interval of Tolerance has been optimized to obtain the smoothest prosthesis surface. In relation to the different discontinuities of CAD model, this optimum (IT $=0.023 \mathrm{~mm}$ ) was deduced from the D.O.E. model. After milling, the area of the real surface where the CAD model produces the greatest $\mathrm{C}^{1}$ discontinuity (0.5788) was characterised by optical measurements. In this zone, the smoothness was evaluated to $0.453 \mathrm{~mm}$ which matches the statistical limits envelop of the response surface $(0.4290 .046 \mathrm{~mm})$.

\section{Conclusion}

This paper deals with the surface quality of machined knee prostheses. A new toolpath computation method has thus been presented. It uses a sliding five degree polynomial interpolation to ensure continuity of the computed toolpath, in both slope and curvature. A D.O.E. has then been conducted to compare the surface quality obtained by the presented method and a classical interpolation using CNC enhancement facilities. Furthermore, a smoothness parameter of the surface has been defined to quantify the ability of an interpolation method to smooth undesired discontinuities. For the three main types of discontinuities $\left(\mathrm{C}^{0}, \mathrm{C}^{1}\right.$ and $\left.\mathrm{C}^{2}\right)$, the polynomial interpolation showed a great improvement of the surface quality. An accurate empirical model has then been defined to optimize the discretisation of the CAD/CAM model and thus improve the surface quality. The method has been tested on a real prosthesis.

\section{References}

[1] Nevelos J (2004) Surface Engineering of Prosthetic Knee Components. The Knee 11(3): 163-167

[2] Ramsden JJ, Allen DM, Stephenson DJ, Alcock JR, Peggs GN, Fuller G, Goch G (2007) The Design and Manufacture of Biomedical Surfaces. CIRP Annals 49(2):687-711.

[3] Lartigue C, Tournier C, Ritou M, Dumur D (2004) High-Performance NC for HSM by Means of Polynomial Trajectories. CIRP Annals 53(1):317-320.

[4] Schützer K, Stroh C, Schulz H (2010) C-Space Based Approach for the Calculation of Toolpaths for Freeform Surfaces in B-Spline Description. CIRP Annals 59(1):421-424.

[5] Altintas Y, Erkorkmaz K (2003) Feedrate Optimization for Spline Interpolation in High Speed Machine Tools. CIRP Annals 52(1):297-302.

[6] Bachmann J, Linares J-M, Sprauel J-M, Bourdet P (2004) Aide in DecisionMaking: Contribution to Uncertainties in Three-Dimensional Measurement Precision Engineering 28(1):78-88. 